\title{
CONCERNING CONTINUOUS SELECTIONS
}

\author{
SAM B. NADLER, JR. AND L. E. WARD, JR. ${ }^{1}$
}

\begin{abstract}
Necessary and sufficient conditions are given in order that certain types of partially ordered continua admit a continuous selection on the hyperspace of nonempty compact connected subsets. We establish that the class of arcwise connected compacta which admit continuous selections on their space of subcontinua is a proper subclass of the dendroids. This class is also shown to be larger than the class of metrizable generalized trees.
\end{abstract}

1. Introduction. In what follows a continuum is a nonempty compact connected Hausdorff space. If $X$ is a topological space, then $2^{X}$ denotes the space of all nonempty closed subsets of $X$, with the Vietoris topology [9]. The subspace of closed and connected subsets of $X$ is denoted $C(X)$. A continuous selection for a family $\alpha \subset 2^{X}$ is a continuous function $\sigma: \alpha \rightarrow X$ such that $\sigma(A) \in A$ for each $A \in \alpha$.

Recently Kuratowski, Nadler, and Young [7] have proved that if $X$ is a metrizable continuum, then a continuous selection for $2^{X}$ exists if and only if $X$ is an arc. If one seeks a continuous selection on $C(X)$, however, then it is known that this can be done if $X$ is a tree [2]. In this note we will prove that the existence of a continuous selection for $C(X)$ is equivalent to the hereditary unicoherence of $X$, provided $X$ is a member of a large class of metrizable continua which includes the Peano continua. Therefore, as a corollary it follows that the dendrites are precisely those Peano continua $X$ for which $C(X)$ admits a continuous selection.

Our proof of these results employs a theorem of Kelley [3] which asserts that if $X$ is a metrizable continuum then $C(X)$ is arcwise connected. The corresponding result for $2^{X}$ is due to Borsuk and Mazurkiewicz [1]. We note that M. M. McWaters [8] has recently extended these theorems to the case of nonmetrizable continua.

\section{A selection theorem.}

Lemma 1. If $S^{1}$ is the unit circle in the complex plane then $C\left(S^{1}\right)$ is a 2-cell.

Received by the editors July 30, 1969 and, in revised form, November 11, 1969. AMS Subject Classifications. Primary 5465; Secondary 5456.

Key Words and Phrases. Dendrite, dendroid, directed space, generalized tree, selection.

1 The second author was supported in part by a grant from the National Science Foundation. 
Proof. Let $K$ be the cone over $S^{1}$ obtained by joining each point of $S^{1}$ to a point $(0,0,2 \pi)$ in 3 -space by a straight line segment. We define $h: C\left(S^{1}\right) \rightarrow K$ by letting $h\left(S^{1}\right)=(0,0,2 \pi)$. If $A$ is a (possibly degenerate) arc of $S^{1}$, let $l$ be the arc length of $A$ and let $a_{0}$ be the point of $A$ which divides $A$ into two subarcs of equal length. If $J$ is the line segment in $K$ which joins $a_{0}$ to $(0,0,2 \pi)$, we define $h(A)$ to be the point on $J$ whose $z$-coordinate is $l$. It is routine to verify that $h$ is a homeomorphism of $C\left(S^{1}\right)$ on to $K$.

Lemma 2. If $S^{1}$ is the unit circle in the complex plane then $C\left(S^{1}\right)$ admits no continuous selection.

Proof. If $\sigma: C\left(S^{1}\right) \rightarrow S^{1}$ is a continuous selection then, by Lemma 1 , $\sigma$ is, in effect, a retraction of a 2-cell onto its boundary. It is well known that no such retraction exists.

Recall that a dendroid is a metrizable continuum which is arcwise connected and hereditarily unicoherent.

LEMMA 3. If $X$ is a metrizable continuum which admits a continuous selection for $C(X)$, then $X$ is a dendroid.

Proof. By a result of Kelley [3], $C(X)$ is arcwise connected, and if there exists a continuous selection $\sigma: C(X) \rightarrow X$ then $X$ is also arcwise connected. If $X$ is not a dendroid then it contains subcontniua $A$ and $B$ such that $A \cap B$ is not connected. Let $x$ and $y$ be points which lie in distinct components of $A \cap B$. Since $A=\sigma(C(A))$ is arcwise connected, $A$ contains an arc $J_{A}$ whose endpoints are $x$ and $y$. Similarly $B$ contains an arc $J_{B}$ whose endpoints are $x$ and $y$. Since $J_{A}-J_{B}$ and $J_{B}-J_{A}$ are nonempty, it follows that $J_{A} \cup J_{B}$ contains a simple closed curve $S$ and therefore $\sigma \mid C(S)$ is a continuous selection, contrary to Lemma 2.

If $\Gamma$ is a partial order on a set $X$ then $\Gamma x=\{y \in X:(y, x) \in \Gamma\}$. By a directed space we mean a topological space $X$ with a closed partial order $\Gamma$ such that the family $\{\Gamma x: x \in X\}$ has the finite intersection property, i.e., if $x_{1}, x_{2}, \cdots, x_{n}$ are elements of $X$ then $\Gamma x_{1} \cap \Gamma x_{2} \cap \cdots$ $\cap \Gamma x_{n}$ is nonempty. A generalized tree is a compact directed space in which each of the sets $\Gamma x$ is an arc and each subcontinuum has a zero. It follows from the results of [10] and [6] that a dendrite is a metrizable generalized tree and that a compact connected directed space is a generalized tree if and only if it is hereditarily unicoherent and each of the sets $\Gamma x$ is connected. Further, if $X$ is a compact metrizable directed space in which each of the sets $\Gamma x$ is connected, then it follows from a result of R. J. Koch [5] or [11] that $X$ is arcwise connected. 
ThEOREM 1. Let $X$ be a compact metrizable directed space and suppose that $\Gamma x$ is connected for each $x \in X$. Then $C(X)$ admits a continuous selection if and only if $X$ is a generalized tree.

Proof. In view of Lemma 3 it suffices to show that if $X$ is a generalized tree then $C(X)$ admits a continuous selection. If $A \in C(X)$ then $A$ has a zero, $z(A)$. We will show that $z$ is continuous. Let $A_{\alpha}$ be a net in $C(X)$ and suppose $A_{\alpha} \rightarrow A$. Since $X$ is compact we may assume that $\lim z\left(A_{\alpha}\right)$ exists. Then $\lim z\left(A_{\alpha}\right) \in A$ and therefore $z(A) \in$ $\Gamma\left(\lim z\left(A_{\alpha}\right)\right)$. On the other hand there are elements $x_{\alpha} \in A_{\alpha}$ such that $\lim x_{\alpha}=z(A)$. Since $z\left(A_{\alpha}\right) \in \Gamma x_{\alpha}$ for each $\alpha$ and since $\Gamma$ is closed, we infer that $\lim z\left(A_{\alpha}\right) \in \Gamma z(A)$. Therefore $\lim z\left(A_{\alpha}\right)=z(A)$ and the theorem is proved.

It has been proved by Virginia Walsh Knight [4] that every Peano continuum is a directed space in which the sets $\Gamma x$ are connected. Therefore the following corollary is immediate.

Corollary. If $X$ is a Peano continuum then $C(X)$ admits a continuous selection if and only if $X$ is a dendrite.

We note that there is a simple direct proof of this corollary. For if $X$ is a Peano continuum and if $C(X)$ admits a continuous selection, then $X$ contains no simple closed curve by Lemma 2 . Thus $X$ is a dendrite. The converse follows from [2].

3. Two examples. It is tempting to conjecture that among the arcwise connected compacta the existence of a continuous selection for the space of subcontinua is equivalent to the property of being a dendroid. Lemma 3 shows that if such a continuous selection exists then the compactum is a dendroid. However, the converse is not true. To see this let $A_{n}$ be the line segment joining $(-1,0)$ and $\left(1,2^{-n}\right)$ in the plane, for $n=0,1,2, \cdots$, and let $T$ be the line serment joining $(-1,0)$ and $(1,0)$. It is apparent that $D_{1}=T \cup_{n=0}^{\infty}\left\{A_{n}\right\}$ is a dendroid.

Lemma 4. If $\sigma$ is a continuous selection on $C\left(D_{1}\right)$ then $\sigma(T)=(-1,0)$.

Proof. For each $n=0,1,2, \cdots$ let $\alpha\left(2^{-n}\right)=A_{n}$. Clearly $\alpha\left(2^{-n}\right) \rightarrow T$ and there is an $\operatorname{arc} B_{n} \subset C\left(D_{1}\right)$ whose endpoints are $\alpha\left(2^{-n-1}\right)$ and $\alpha\left(2^{-n}\right)$ and such that each member of $B_{n}$ contains either $A_{n+1}$ or $A_{n}$. We extend $\alpha$ to each segment $\left[2^{-n-1}, 2^{-n}\right]$ as a homeomorphism onto $B_{n}$. Then for any sequence $t_{n} \rightarrow 0$ it is clear that $\alpha\left(t_{n}\right) \rightarrow T$, and hence if we let $\alpha(0)=T$ then $\alpha$ defines an arc in $C\left(D_{1}\right)$. Now suppose $\sigma(T)=(t, 0)$ with $t>-1$. Since $\sigma \alpha([0,1])$ is locally connected, it follows that 
$\sigma \alpha\left(2^{-n}\right)=\left(t_{n}, 0\right)$ with $t_{n}>-1$, for sufficiently large $n$. But then $\sigma \alpha\left(2^{-n}\right) \notin \alpha\left(2^{-n}\right)$ which is contrary to the hypothesis that $\sigma$ is a selection.

TheORem 2. There exists a dendroid $D$ such that $C(D)$ admits no continuous selection.

Proof. Let $D=D_{1} \cup D_{2}$ where $D_{2}$ is the reflection of $D_{1}$ about the origin (see Figure 1). Suppose there exists a continuous selection $\sigma: C(D) \rightarrow D$. Then $\sigma_{1}=\sigma \mid C\left(D_{1}\right)$ and $\sigma_{2}=\sigma \mid C\left(D_{2}\right)$ are also continuous selections. By Lemma $4, \sigma(T)=\sigma_{1}(T)=(-1,0)$ and $\sigma(T)=\sigma_{2}(T)$ $=(1,0)$, which is impossible.

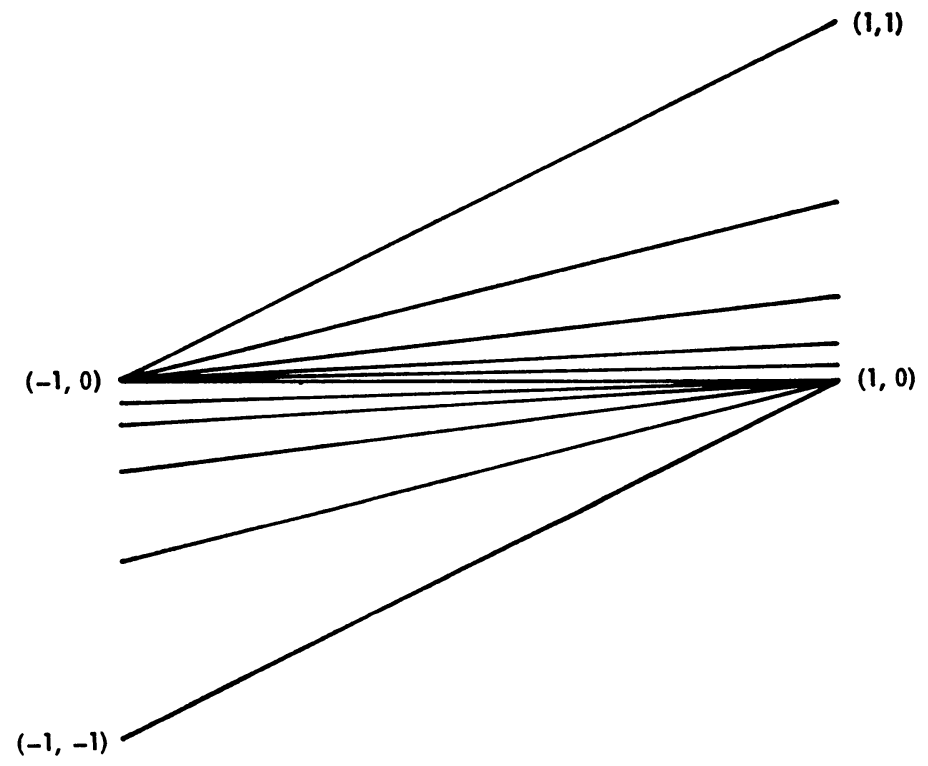

FIGURE 1

We have established that the class of arcwise connected compacta which admit continuous selections on the space of subcontinua is a proper subclass of the dendroids. The next example shows that this class is larger than the class of metrizable generalized trees. The example was first exhibited by Koch and Krule [6] who asserted that it is a dendroid which is not a generalized tree.

THEOREM 3. There exists a dendroid $D$ which cannot be partially ordered as a generalized tree and which admits a continuous selection $\sigma: C(D) \rightarrow D$. 
Proof. In the plane let $A=\{(x, 2): 0 \leqq x \leqq 1\}$, let $T=\{(0, y): 0$ $\leqq y \leqq 2\}$, and for each $n=0,1,2, \cdots$ let $A_{n}=\left\{\left(2^{-n}, y\right): 1 \leqq y \leqq 2\right\}$. Let $D_{1}=A \cup T \cup \cup_{n=0}^{\infty}\left\{A_{n}\right\}$, let $D_{2}$ be the reflection of $D_{1}$ about the $x$-axis and let $D=D_{1} \cup D_{2}$ (see Figure 2).

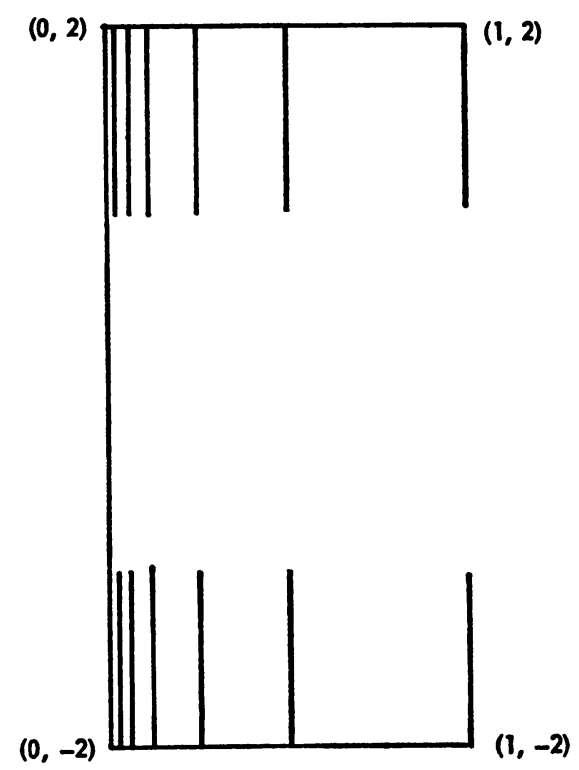

FIgURE 2

If $D$ can be partially ordered as a generalized tree, with partial order $\leqq$, then $D$ has a zero, 0 . Without loss of generality we suppose $0 \in D_{2}$. Then for each $n=0,1,2, \cdots$ we see that $\left(2^{-n}, 2\right)<\left(2^{-n}, 1\right)$ and that $(0,1)<(0,2)$, since for each $x \in D$ the set $\{y: y \leqq x\}$ must be an arc. However $\left(2^{-n}, 2\right) \rightarrow(0,2)$ and $\left(2^{-n}, 1\right) \rightarrow(0,1)$ so that if the partial order is closed then it must be that $(0,2) \leqq(0,1)$. Therefore $D$ is not a generalized tree.

We shall now define a continuous selection on $C(D)$. Note first that $D_{1}$ is a generalized tree when $0=(0,2)$, and also $D_{2}$ is a generalized tree when $0=(0,-2)$. Hence if $\sigma_{i}: C\left(D_{i}\right) \rightarrow D_{i}(i=1,2)$ is defined by $\sigma_{i}(A)=$ the zero of $A$ relative to the natural partial order, then $\sigma_{i}$ is a continuous selection. Moreover, by an argument similar to that of Lemma $4, \sigma_{1}(T)=(0,2)$ and $\sigma_{2}\left(T^{\prime}\right)=(0,-2)$, where $T^{\prime}$ is the reflection of $T$ about the $x$-axis. For each $A \in C\left(T \cup T^{\prime}\right)$ let $\phi_{1}(A)$ be the second coordinate of $\sigma_{1}(\mathrm{~A} \cap T)$ if $A \cap T \neq \varnothing$, and otherwise let $\phi_{1}(A)$ $=0$. Similarly, let $\phi_{2}(A)$ be the second coordinate of $\sigma_{2}\left(A \cap T^{\prime}\right)$ if $A \cap T^{\prime} \neq \varnothing$, and otherwise let $\phi_{2}(A)=0$. Now define 


$$
\tilde{\sigma}(A)=\left(0, \phi_{1}(A)+\phi_{2}(A)\right)
$$

for $A \in C\left(T \cup T^{\prime}\right)$, and let $\tilde{\sigma}=\sigma_{i}$ on $C\left(D_{i}\right)$. Then $\tilde{\sigma}$ is a continuous selection on $C\left(D_{1}\right) \cup C\left(D_{2}\right) \cup C\left(T \cup T^{\prime}\right)$. The extension of $\tilde{\sigma}$ to a continuous selection $\sigma$ on $C(D)$ is now straightforward. If $A \in C(D)$ $-\left(C\left(D_{1}\right) \cup C\left(D_{2}\right) \cup C\left(T \cup T^{\prime}\right)\right)$ define

$$
\sigma(A)=\tilde{\sigma}\left(A \cap\left(T \cup T^{\prime}\right)\right) .
$$

\section{REFERENCES}

1. K. Borsuk and S. Mazurkiewicz, Sur l'hyperspace d'un continu, C. R. Sci. Varsovie 24 (1931), 149-152.

2. C. E. Capel and W. L. Strother, Multi-valued functions and partial order, Portugal. Math. 17 (1958), 41-47. MR 21 \#322.

3. J. L. Kelley, Hyperspaces of a continuum, Trans. Amer. Math. Soc. 52 (1942), 22-36. MR 3, 315.

4. Virginia Walsh Knight, A continuous partial order for Peano continua, Pacific J. Math. 30 (1969), 141-154.

5. R. J. Koch, Arcs in partially ordered spaces, Pacific J. Math. 9 (1959), 723-728. MR $21 \# 7269$.

6. R. J. Koch and I. S. Krule, Weak cutpoint ordering on hereditarily unicoherent continua, Proc. Amer. Math. Soc. 11 (1960), 679-681. MR 22 \#11356.

7. K. Kuratowski, Sam B. Nadler, Jr. and G. S. Young, Continuous selections on locally compact separable metric spaces, Bull. Acad. Polon. Sci. (to appear).

8. M. M. McWaters, Arcs, semigroups, and hyperspaces, Canad. J. Math. 20 (1968), 1207-1210. MR 37 \#6914.

9. E. Michael, Topologies on spaces of subsets, Trans. Amer. Math. Soc. 71 (1951), 152-182. MR 13, 54.

10. L. E. Ward, Jr., Mobs, trees and fixed points, Proc. Amer. Math. Soc. 8 (1957), 798-804. MR 20 \#3516.

11. - Concerning Koch's theorem on the existence of arcs, Pacific J. Math. 15 (1965), 347-355. MR 31 \#6206.

State University of New York at Buffalo, Buffalo, New York 14226

Dalhousie University, Nova Scotia, Canada and

University of Oregon, Eugene, Oregon 97403 